\title{
Effects of learning on judgment in the presence of discrepant anchors
}

BERTRAM L. KOSLIN AND RICHARD PARGAMENT, PRINCETON UNIVERSITY STUART LEVINE, BARD COLLEGE

Assimilation and contrast shifts were obtained in an anchor discrepancy by learning design. Ss with a better learned judgment scale shifted less than Ss who had not stabilized a scale. Extremely discrepant anchors became somewhat irrelevant to Ss' judgment. Irrelevancy was manifested by a diminution of the contrast shift.

In many judgment studies, shift effects have been obtained by interpolating an anchor (standard) into a previously judged stimulus series. The shifts have generally been in the direction away from the anchor (contrast effect), but some studies report shifts toward the anchor (assimilation effect) when the anchoring stimulus is contiguous with the judged stimuli. Contrast results from the formation of a new judgment scale which includes the learned stimuli and the introduced anchor. Previously judged stimuli are given new scale values, thereby permitting the integration of the discrepant stimulus. Assimilation is the tendency to cluster somewhat discrepant stimuli with that which has already been learned (Sherif \& Hovland, 1961).

Individuals with more stabilized scales have generally expended greater efforts in learning, and are more likely to attempt retaining their scales when novel or discrepant information is introduced. Individuals who have stabilized scales should therefore contrast their judgments to a lesser extent than those with unstable scales. Thus, when anchors become sufficiently discrepant, making it difficult to cluster them with what has already been learned, contrast shifts generally result. However, Ss who have stabilized a judgment scale should integrate anchors in a manner which is not disruptive to that scale; there should be less of a change in judgment for antecedently learned stimuli.

When introduced anchors are extremely remote from previously learned stimuli, they may be perceived as being irrelevant to the judgment task and therefore ignored (Bevan \& Darby, 1955). Since contrast indicates a change in judgment organization to incorporate the anchor, anchors found irrelevant would not be incorporated and should produce less contrast. Because individuals who have more effectively scaled an initial series of stimuli are better able to evaluate the extent of the difference between learned stimuli and extremely remote anchors, a narrower range of anchov discrepancies should be perceived as being relevant to the judgment task. Therefore, a diminution of the contrast shift should occur at smaller discrepancies for Ss with more stable scales.

\section{Method}

Ss judged a series of weights which were re-presented with an anchor introduced. As the relevance of anchor discrepancy was of concern, it was important to introduce anchors in a manner which did not force Ss to integrate anchors into their scales for judgment. Anchors remained unjudged by Ss and were not labelled by $\mathrm{E}$. A technique used by White (1964) of adding new numerical categories for anchor sessions also avoided forced integration, and corrected for the end effects occurring with restricted response languages (Webb \& Bevan, 1958).

Apparatus and Stimulus Series. A vertical board obstructed Ss $^{\prime}$ view of E. Ss pulled down a handle attached to a wire (suspending the weights) which ran through the board to $\mathrm{E}$ over low friction pulleys.

The weight values were drawn from the region where JND decrements are represented by a relatively constant fraction $(\mathrm{SD} / \mathrm{S}=.0325) .1$ Five weights $(280,332$, 398,480 , and $580 \mathrm{~g}$ ) equally spaced over 24 JNDs constituted the learning series. The anchors ranged from 580 to $3070 \mathrm{~g}$.

Procedure. College age Ss ordered the weights from low to high by assigning them numbers from 6 through 10. The weights were randomized within blocks, and presented by the method of single stimuli for 100 trials. Each $S$ then received one of seven anchor treatments. Ss were told that they would not judge every lifted weight. When a judgment was required, a light was flashed prior to lifting the weight. Ss judged (the original five weights) by the method of single stimuli using an expanded category system (1 through 15). The five judged stimuli were each lifted six times and the anchor 30 times.

Learning Criteria. Ss were divided into high learners (HL) and low learners (LL). The criteria defining HL were: (a) at least $50 \%$ correct responses, and (b) less than a $20 \%$ reduction in correct responses from the first to the last 20 acquisition trials. Ss not meeting these criteria were the LL. There were $70 \mathrm{HL}$ and 72 LL Ss.

\section{Results}

A learning by anchor discrepancy by individual weight analysis of covariance is summarized in 1 able 1. The learning session means were subtracted from their respective covariance adjusted scores to obtain the shift suores plotted in Fig. 1. The LL contrasted their judgments to a greater extent than did 
the HL. Of course, an anchor treatments main effect was obtained. Principally because the weight means were further apart in many anchor treatments than in the learning session, a main effect due to weights resulted. The anchor treatment by weight interaction showed that each weight was differentially affected by its particular distance from the anchors.

The shift curves for the LL suggest a slight diminution of contrast, and the curves for the HL indicate a somewhat greater diminution. However, trend tests indicated that only the linear component was significant $(F=7.85$, df $=1 / 127, p<.01)$.

Since the adjusted shift scores resulted from changes in skewness of the judgment distribution and the extent to which Ss used new categories, additional procedures measured category shift independent of skew. The end categories used in the anchor session were compared with categories 6 and 10, the end categories of the learning session. The number of categories used below 6 was subtracted from the number of categories used above 10. Thus positive scores (assimilation) and negative scores (contrast) reflected the degree to which $\mathrm{Ss}$ used additional categories above and below the original response scale. A Freeman-Tukey square root transform stabilized the variance of the category shift scores. Analysis of variance demonstrated that anchor treatments produced significant differences in the usage of new categories $(F=9.00, d f=6 / 128, p<.005)$. Trend analyses for the anchor discrepancy profile revealed linear $(F=48.62, d f=1 / 135, p<.005)$ and quadratic components $(\mathrm{F}=7.25, \mathrm{df}=1 / 135, \mathrm{p}<.01)$.

\section{Discussion}

In the contrast range, the HL did not shift from their first organizations to the extent that the $L L$ shifted when discrepant anchors were introduced. It thus seems reasonable to conclude that the HL were attempting to retain their initial scalar organizations to a greater extent. Moreover, the data do not suggest that the HL would have shifted their judgments further over more trials as in Tresselt's (1947) research.

Under conditions which did not oblige Ss to place the anchor into their response scale, contrast di-

Table 1. Covariance Analysis of Mean Category Scores

\begin{tabular}{lrrr} 
Source & df & $M S^{\prime}$ & $F$ \\
\hline Between Subjects & & & \\
Learning (A) & 1 & 98.81 & $20.42^{*}$ \\
Anchor (B) & 6 & 45.85 & $9.47^{*}$ \\
AB & 6 & 1.34 & $<1$ \\
Error (b) & 127 & 4.84 & \\
Within Subjects & & & $71.44 *$ \\
Weights (C) & 4 & 31.29 & 2.53 \\
AC & 4 & 1.11 & $7.28^{*}$ \\
BC & 24 & 3.19 & $<1$ \\
ABC & 24 & .38 & \\
Error (w) & 511 & .44 & \\
\hline
\end{tabular}

$* p<.005$

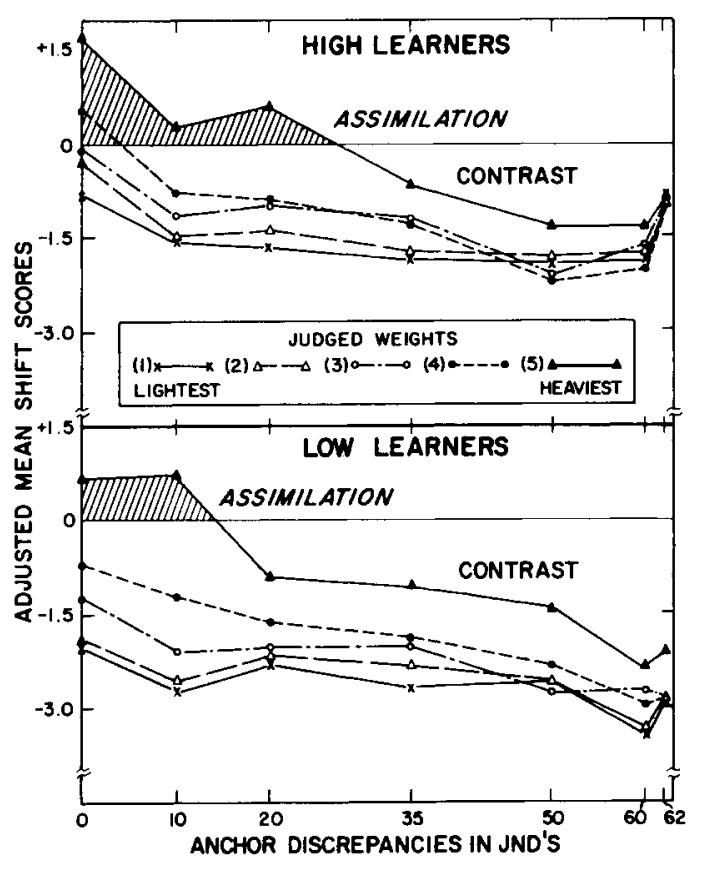

Fig. 1. Mean category shift as a function of anchor discrepancy.

minished indicating that remote anchors may become irrelevant. Assimilation-contrast category shift proved to be a curvilinear function of anchor discrepancy. Since no learning level by anchor discrepancy interaction resulted, it was not possible to conclude that there was a greater diminution of contrast for the HL than for the LL, although the means fall in the predicted direction.

The results suggest that assimilation-contrast is best described by a "lopsided sine wave." As anchor discrepancy increases, assimilation attains a maximum and then declines. At higher anchor discrepancies, contrast attains a maximum and then declines so that at extreme discrepancies, no shift effects areobtained.

\section{References}

BEVAN, W., \& DARBY, C. L. Patterns of experience and the constancy of an indifference point for perceived weight. Amer. J. Psychol., 1955, $68,575-584$.

SHERIF, M., \& HOVLAND, C. I. Social judgment. New Haven: Yale University Press, 1961.

TRESSELT, MARGARET E. The influence of amount of practice upon the formation of a scale of judgment. J. exp. Psychol., 1947, 37, 251-260.

WEBB, S. C., \& BEVAN, W. Single standard judgment: A reorientation in rating scale procedure. J. gen. Psychol., 1958, 59, 119-133.

WHITE, B. J. Availability of categories and contrast-effects in judgment. Amer. J. Psychol., 1964, 77, 231-239.

WOODWORTH, R. S. Experimental psychology. New York: Henry Holt, 1938.

Note

1. The mass of the weights was derived by interpolating from the empirical data of several studies (Woodworth, 1938). The origin $(280 \mathrm{~g})$ was chosen so that none of the judged weights corresponded to natural standards (e.g., a pound). 